\title{
Des modèles utiles mais ignorés: le cas des relations publiques
}

\section{Bernard Dagenais}

\section{(2) OpenEdition}

1 Journals

Édition électronique

URL : http://journals.openedition.org/communicationorganisation/3463

DOI : 10.4000/communicationorganisation.3463

ISSN : $1775-3546$

Éditeur

Presses universitaires de Bordeaux

\section{Édition imprimée}

Date de publication : 1 octobre 2006

Pagination : 148-162

ISSN : 1168-5549

\section{Référence électronique}

Bernard Dagenais, "Des modèles utiles mais ignorés: le cas des relations publiques », Communication et organisation [En ligne], 30 | 2006, mis en ligne le 21 juin 2012, consulté le 19 avril 2019. URL : http:// journals.openedition.org/communicationorganisation/3463 ; DOI : 10.4000/

communicationorganisation.3463

Ce document a été généré automatiquement le 19 avril 2019

(C) Presses universitaires de Bordeaux 


\title{
Des modèles utiles mais ignorés: le cas des relations publiques
}

\author{
Bernard Dagenais
}

1 Les sciences humaines ont souvent emprunté aux sciences dites exactes les différents modèles qui ont inspiré leur discipline. La théorie politique de Marx sur la lutte des classes a puisé à même la théorie de l'évolution des espèces de Darwin à qui Marx avait dédié son livre Le Capital.

Dans l'univers des communications, c'est le modèle mathématique de Shannon et Weaver ${ }^{1}$ qui a donné naissance au paradigme de la communication: $\mathrm{E}-\mathrm{M}-\mathrm{R}$ (émetteur message- récepteur). Ce sont les recherches sur la transmission technique des messages qui ont permis de densifier ce paradigme en tenant compte d'une part des bruits pouvant perturber la réception (des parasites dans la ligne de transmission) et d'autre part du feedback (des réponses pouvant influencer le message comme dans le cas classique des thermostats).

3 Les sciences sociales ont également fourni aux communications plusieurs modèles utiles. Déjà en 1948, le politicologue Laswell ${ }^{2}$ avait défini l'acte de communication dans la formule devenue classique : Qui, dit quoi, à travers quel canal, à qui et pourquoi. Quelques années plus tard, la théorie du two step flow des politicologues Katz et Lazarsfeld ${ }^{3}$ démontrait l'importance des leaders d'opinion dans la transmission des messages et l'importance des échanges interpersonnels.

On constate par ailleurs une résistance de certaines écoles de pensée à accepter des modèles qui ne cadrent pas avec leur culture scientifique. En communication, le monde anglo-saxon, par exemple, a été peu enclin à intégrer des modèles dits culturels. Alors que la théorie de la dynamique de la culture d'Abraham Moles ${ }^{4}$ était développée dès 1967, McQuail et Windahl, dans leur livre classique sur les modèles de communication, datant de 1981, n'en font aucune mention. Par ailleurs, Willett ${ }^{5}$, de culture francophone, consacre à ce modèle, le même espace qu'aux classiques anglophones.

5 Par contre, le monde francophone, qui s'est laissé bercer par les recherches scientifiques sur la génétique de Jacob et Monod $^{6}$, a été hermétique à la transposition qu'Edgar Morin ${ }^{7}$ 
en a faite sur le plan de la genèse et de la circulation de l'information et des idées. Morin a en effet dressé un heureux parallèle entre les découvertes sur l'ADN et le développement de l'embryon et la reproduction du système social en empruntant les concepts d'ordre et de désordre, d'échanges d'information et de contraintes, de la mémoire du tout par chacune des parties et du rôle du milieu ambiant et de l'environnement. Ce modèle, que l'on pourrait appeler génétique, a été aussi ignoré du milieu anglo-saxon. Il existe donc des effets de mode dans les modèles utilisés.

\section{La modélisation}

6 Pourquoi faut-il créer des modèles? Pour Willett ${ }^{8}$, "Chaque personne construit ses propres modèles de perception et de jugement à partir de ses réflexions, de ses observations et de ses expériences de vie. Ces modèles, qu'on utilise chaque jour, ne sont pas très rigoureux, d'un point de vie scientifique, bien qu'ils acquièrent un certain degré de systématisation comme, par exemple, dans le cas des stéréotypes».

7 Et il poursuit: «... lorsque l'on traite de la communication, on peut partir du constat suivant: dans toute société circulent des symboles et des messages qui sont produits, distribués et consommés par l'intermédiaire de certaines personnes, de certains moyens techniques, de certaines entreprises et de certaines institutions sociales. Les modèles qu'on peut énoncer à ce propos servent donc aussi bien à interroger, à analyser, à comprendre et à expliquer qu'à révéler divers aspects de la circulation des messages et des symboles dans une société». Pour cet auteur, comme pour tant d'autres, le modèle sert toutefois davantage à bien comprendre qu'à mieux interagir. Dès lors, la construction d'un modèle s'articulera autour d'une préoccupation de connaissance plutôt que d'un désir de mieux faire. "Le but de la modélisation en science est de faire ressortir et de rendre visibles les principaux aspects ou éléments d'une structure, d'un processus, d'un système ou d'un phénomène faisant l'objet d'une analyse».

8 L'auteur explique le cheminement qui mène à la création d'un modèle. Tout part d'abord d'un concept. Or, pour l'auteur, en communication, "plusieurs concepts, comme l'information, le récepteur, l'auditoire, appartiennent au langage courant et, bien qu'ils aient été l'objet d'un effort de définition rigoureuse à des fins d'analyse, ils sont souvent source de beaucoup de confusion quant à la compréhension du phénomène de la communication».

Du concept scientifique découle la théorie. Et le modèle se situe dans le prolongement d'une théorie. Le modèle apparaît «comme une partie concrète de la théorie qui est directement en rapport avec un ensemble de comportements. Dans cette optique, il permet d'avoir prise techniquement sur la réalité». Willett parle ultimement de quatre types de modèles : le modèle cognitif, le modèle prévisionnel, le modèle décisionnel et le modèle normatif. Si le modèle peut ultimement avoir une certaine utilité, on aura compris que son existence est heuristique avant d'être pratique. Pour les communicologues, cela ne cause aucun problème. Pour les communicateurs appelés à réaliser des stratégies concrètes de communication, les modèles demeurent trop abstraits ou demandent trop d'énergie pour être utiles. Ainsi, en communication, les modèles pensés par les scientifiques remplissent bien la définition qu'en donne Willett, c'est-àdire cherchent à comprendre et à expliquer les phénomènes de la communication, mais servent mal les professionnels de la communication. Il ne faut pas voir dans ce constat une critique de l'approche scientifique, ou un malaise sur la façon dont les professionnels 
travaillent. Il s'agit plutôt de réaliser la difficulté de construire, en communication, des modèles capables de satisfaire les chercheurs comme les praticiens. Nous allons illustrer ce constat à travers la pratique des relations publiques.

\section{Une définition des relations publiques}

10 Le terme de relations publiques n'a pas la même connotation partout, même si la littérature en donne la même définition des deux côtés de l'atlantique. En France certains professionnels de la communication cantonnent les relations publiques dans le hors média ou parfois dans les seules relations de presse. Les relations publiques touchent certes ces deux secteurs, mais englobent plus grand. Des auteurs classiques comme Lougovoy et Huisman ${ }^{9}$ en France, ou Cutlip, Center et Broom ${ }^{10}$ aux États-Unis, partagent la même approche des relations publiques. La définition qui fait consensus est celle de l'International Public Relations Association (IPRA), reprise et modulée par les diverses associations nationales de relations publiques :

Public Relations practice is the planned and sustained effort to establish and maintain goodwill and mutual understanding between an organization and its publics.

11 La pratique des relations publiques consiste donc à investir des efforts planifiés et permanents pour établir et maintenir des rapports de bonne volonté et de compréhension mutuelle entre une organisation et ses publics. Dans ce sens, les relations publiques sont synonymes de communication des organisations et englobent autant les communications internes qu'externes, la communication d'image et corporate et sont utilisées pour faire connaitre, pour favoriser une attitude positive et pour provoquer des comportements attendus du citoyen/consommateur.

\section{Les buts poursuivis par les relations publiques}

Que recherchent les relations publiques? D'abord et avant tout, mettre en valeur l'organisation, l'entreprise, l'individu qui les sollicitent. Il s'agit donc d'une entreprise de séduction, de conviction et fondamentalement d'influence. Elles doivent accompagner l'entreprise dans la gestion des enjeux et des crises, dans la mise en marché de ses produits, services et idées, dans la définition et la défense de son image. De ce fait, les relations publiques doivent construire une certaine réalité, forger une opinion publique favorable ou contrer une opinion défavorable, agir sur le présent et le futur et non plus uniquement analyser le passé.

Les relations publiques doivent affronter sur la place publique des concurrents et des adversaires, partager la sphère publique avec des partenaires sympathiques et des groupes hostiles, rencontrer tous les jours les journalistes dans leur quête de nouvelles qui leur semblent d'autant plus intéressantes qu'elles sont alimentées par la controverse, le scandale, le soit disant faux-pas.

14 Pour interagir avec la réalité, les modèles dont elles auront recours doivent viser le résultat et non l'analyse. Noelle-Neumann ${ }^{11}$ décrit de façon fort intéressante le phénomène de la spirale du silence mais n'explique ni comment l'affronter, ni comment la prévenir, ni comment la désamorcer. Il en est de même des autres théories que l'on retrouve en relations publiques dont nous parlerons plus loin. 
Puisque nous allons mettre en parallèle les approches des relations publiques avec celles de la publicité, il serait utile de donner quelques caractéristiques particulières de cet autre métier des communications. Essentiellement la publicité cherche à convaincre le citoyen/consommateur d'acheter un produit ou service, d'adhérer à une idée ou de donner son appui à un individu. Il s'agit donc ici aussi de chercher à influencer la cible visée de façon la plus efficace possible.

Les deux métiers partagent donc les mêmes objectifs, soit faire connaitre, faire apprécier et faire vendre, mais elles fonctionnent selon des modes tout à fait différents. Les relations publiques essaient de faire endosser ou entériner par une tierce partie les informations qu'elles veulent faire circuler. Par exemple, elles utilisent à cet effet, les relations de presse par lesquelles les journalistes vont rapporter les propos qui leur sont transmis. Cette approche a comme corollaire que l'entreprise ne contrôle pas l'information qu'elle diffuse car le journaliste est libre de répercuter les informations qu'il reçoit et peut très bien les dénigrer comme les endosser.

17 La publicité fonctionne selon un autre registre. L'entreprise prépare elle-même les messages qu'elle veut diffuser, les dispose de la façon qu'elle le souhaite, lui ajoute du son, de l'image, du mouvement comme bon lui semble et réserve un espace dans le média de son choix, dans la plage horaire ou le cahier désiré et paie pour ce faire. De ce fait, elle contrôle absolument le message et le canal par lequel il sera diffusé.

Même si ces deux métiers partagent les mêmes visées, l'entreprise, parce qu'elle doit investir des fonds importants en publicité, alors qu'en relations publiques tout est (relativement) gratuit, va se munir d'outils, d'approches, de modèles bien précis pour s'assurer que ses investissements en publicité sont bien orientés. Nous en reparlerons un peu plus loin.

\section{Les modèles utilisés en relations publiques}

9 Les relations publiques ont développé quelques méthodes et modèles qui leur sont propres. En voici quelques-uns.

\section{RACE}

20 La méthode classique pour réaliser une stratégie de communication s'appelle RACE et a été élaborée en 1963 par Marston ${ }^{12}$. RACE résume les quatre phases du cycle de la production d'un plan de communication: Recherche, Action, Communication et $\underline{E}$ valuation. Cultip et Center parle plutôt des fonctions types, soit la Découverte des faits, la Planification du programme, la Réalisation du programme et l'Évaluation.

\section{Grunig}

21 Grunig $^{13}$ a décrit quatre modèles de relations publiques : la promotion, l'information publique, la communication bidirectionnelle asymétrique et la communication bidirectionnelle symétrique. Ces quatre modèles tentent de circonscrire l'importance du récepteur du message dans les communications. Le modèle de la promotion consiste à diffuser une information sans se soucier du public. On lui impose un discours de façon unidirectionnelle, sans se demander si celui-ci est exact. Ce que recherche l'émetteur est 
d'imposer son message. Le modèle de l'information publique est toujours une communication unidirectionnelle de la source vers le public visé, mais cette fois, l'émetteur se préoccupe au premier degré de la véracité de ses propos et de l'intérêt du public. Le modèle de la communication bidirectionnelle asymétrique décrit le processus par lequel la source essaie d'adapter son message à son public et prend même le soin de le sonder, sans toutefois prendre l'engagement de répondre à ses besoins. Le modèle de la communication bidirectionnelle symétrique représente la situation idéale en communication où la source non seulement écoute le public, mais tient compte de ses remarques et s'adapte à ses besoins. Nous sommes ici dans un monde idéal peu réaliste compte tenu que nous avons défini plus haut les relations publiques comme un instrument d'influence et non de partage réciproque.

Kugler ${ }^{14}$, dans son livre intitulé Des campagnes de communication réussies, analyse quelque 40 études de cas primés en relations publiques à partir de trois approches théoriques. Elle utilise d'abord Grunig, mais elle s'appuie aussi sur la théorie des réseaux de Lavigne et sur la théorie des contextes de communication de Mucchielli.

\section{Les réseaux}

Pour Lavigne ${ }^{15}$, on ne doit plus penser la communication de façon linéaire où une source cherche à séduire ou convaincre un public cible, mais davantage en terme de réseaux de communication. Il présente trois grands types de réseaux: les réseaux de liens, les réseaux de contrôles et ceux de transactions. Les réseaux de liens s'appuient sur les relations humaines qu'entretiennent les individus entre eux de manière informelle et conviviale. Les réseaux de contrôles sont caractérisés par un échange économique entre des partenaires qui peuvent exercer un contrôle les uns sur les autres. Les réseaux de transactions reposent sur des activités utilitaires poursuivies par les partenaires avec des approches adaptées à chacun d'eux. Cette théorie des réseaux est intéressante parce qu'elle mêle relations et intérêts entre des partenaires et non plus des relations de domination. Il s'agit d'une approche systémique. Toutefois, cette théorie est difficile à utiliser parce que les trois modèles se retrouvent très souvent dans de multiples échanges. Son intérêt pour le praticien est d'insister sur le fait que les médias ne sont pas les seuls canaux de communication et que les réseaux peuvent exercer un rôle prépondérant dans une stratégie de relations publiques.

\section{Le contexte}

Mucchielli ${ }^{16}$ ajoute une autre dimension. Ce qui importe, ce n'est plus seulement la qualité $\mathrm{du}$ rapport entre la source et la cible prônée par Grunig, ni la richesse des réseaux dans lesquels interviennent les acteurs décrite par Lavigne, mais le contexte dans lequel intervient l'échange. Mucchielli insiste sur quatre contextes: le contexte des positions respectives des acteurs, le contexte relationnel social immédiat, le contexte culturel de référence aux normes et aux règles, le contexte expressif des identités des acteurs. Ce sont là tous des modèles intéressants pour comprendre les circuits de la communication mais aucun d'eux n'est fonctionnel. Ce sont des modèles qui tentent d'expliquer ce qui se passe, mais de façon passive. Ils ne proposent pas des pistes d'action. En somme, le modèle sert son auteur, mais est de peu d'utilité à l'acteur. Aucun professionnel des 
communications ne s'impose en affichant le recours à l'une ou l'autre de ces approches théoriques.

\section{Le temps et l'espace}

Philibert ${ }^{17}$ a essayé de définir un modèle qui permettrait au communicateur de mieux déterminer ses activités prioritaires dans l'exercice quotidien de sa profession. Ce modèle a été construit autour des notions d'espace et de temps. Selon lui, « le principe logique (d') une communication ne peut avoir lieu en dehors d'un temps et d'un espace» ${ }^{18}$ qu'une organisation partage avec ses publics au quotidien.

26 Et « l'expression quotidienne de l'espace et du temps se manifeste à travers les concepts d'importance et d'urgence d'une action.», idée qu'il emprunte à Stephen R. Covey ${ }^{19}$. Pour celui-ci, pour arrêter ce qui est prioritaire à faire, il faut évaluer si une action est urgente ou non et importante ou non. En fait les actions de tout individu oscillent entre des activités non importantes, mais jugées urgentes, et des actions importantes jugées non urgentes. Ainsi, alors qu'un professionnel travaille à une tâche éminemment importante, la réception d'un appel téléphonique d'un supérieur souhaitant voir le professionnel sur le champ pour lui demander un avis sur une action tout à fait secondaire, crée une urgence sans importance. Alors qu'une crise qui met en péril l'organisation est urgente et importante. À l'inverse, il y a des activités importantes, mais non urgentes, comme pratiquer la prévention. Et il y a des activités non urgentes et non importantes, mais tout de même intéressantes comme aller au restaurant avec des clients.

Philibert fait ensuite la démonstration qu'une priorité s'établit de la façon suivante :

IMPORTANCE X URGENCE $=$ PRIORITÉ.

Il fait ensuite un parallèle avec la théorie de la relativité d'Einstein où « la gravitation est une propriété de l'espace/temps sous l'action des masses matérielles. Dans la théorie ... sur l'univers de sens, la priorité est une propriété de l'importance-urgence, qui se transforme sous l'action de la masse des activités de relations publiques» ${ }^{20}$. L'auteur trace alors un parallèle entre la masse, le volume et la distance des actions de relations publiques en ayant recours aux concepts de nécessité, de cohérence, de conviction.

Il termine ainsi son exposé : « Puisque le sens des actions se développe à partir de l'espace et du temps de rencontre avec ces publics, il est primordial de savoir gérer ces deux paramètres» ${ }^{21}$.

Ce qui est intéressant dans cette approche, c'est que l'auteur a organisé autour de concepts très théoriques, un modèle pratique visant à aider les professionnels à faire de meilleurs choix dans leur organisation du temps. L'auteur était parti du principe qu'en relations publiques, tout le monde semble travailler en urgence. Toutefois, ses formules mathématiques étant fort complexes et difficiles à compléter, ce modèle pratique reste ... très théorique.

Le problème de la définition de l'urgence et de l'importance reste entier. En fait, qu'est-ce qu'une priorité ? Est-ce la somme raisonnée du poids d'une action en termes d'urgence ou d'importance ? Mais comment et qui va décider du poids urgence/importance à donner à chaque action. Philibert propose des grilles de pointage intéressantes. Mais on se plait à dire qu'une priorité, c'est une urgence que chacun se fixe. Et une urgence, c'est la priorité d'un autre qui nous est imposée. 
us les modèles décrits plus haut, aucun n'a été construit par des praticiens. Ce sont tous des universitaires, sans expérience sur le terrain, qui les ont élaborés. Et c'est à cause de la rationalité de ces modèles que ces scientifiques ont ensuite été appelés à conseiller les praticiens...

\section{Un modèle informatique}

Une compagnie suisse, Digital Management AG, a développé un logiciel intitulé ComProPractitioner, the software solution for Communication practitioners, avec lequel il est possible de construire une stratégie de communication. Ce logiciel guide le professionnel dans sa démarche lui indiquant les éléments à prendre en considération à chacune des étapes du plan de communication et en livrant des informations sur le bien-fondé de chacune d'entre elles.

À l'instar de tous les logiciels de service, celui-ci dirige l'utilisateur dans les principaux couloirs de la construction d'un plan de communication. Il s'agit là d'un modèle mécanique, très pratique, qui aide le professionnel à suivre un parcours sans faute dans la réalisation d'un plan de communication, mais qui est de peu d'utilité au néophyte. Car le logiciel, à l'instar des panneaux de signalisation routière, confirme à celui qui connaît son chemin, qu'il est toujours dans la bonne direction, mais est incapable de diriger celui qui cherche son chemin.

\section{Un modèle d'évaluation}

L'industrie canadienne des relations publiques s'est ralliée autour d'une nouvelle norme de mesure d'efficacité des campagnes de relations de presse qui se veut l'équivalent en relations publiques des Points d'exposition brut (PEB) de la publicité.

Élaboré par la Société canadienne des relations publiques (SCRP) et endossé par l' Association internationale des professionnels de la communication (AIPC), le système des «points d'évaluation des relations médias» (PEM), disponible sur le site www.mrpdata.com, permet d'évaluer tout type de couverture de presse.

Si les PEM savent évaluer l'intérêt des journalistes pour un objet ou un sujet particulier, ils ne livrent aucune information sur l'impact qu'a pu avoir cette information sur le public. Or, le but poursuivi en relations publiques est d'exercer une influence sur un public donné, et non uniquement de faire circuler une information.

Ainsi donc, en relations publiques, les scientifiques proposent des modèles théoriques et les professionnels des modèles pratiques. Mais peu de modèles proposés ne permettent de mieux interagir pour construire le futur. Le modèle de Philibert est complexe et repose sur une grille compliquée et dissuasive, quoiqu'il permette de poser des jugements pertinents sur l'importance et l'urgence des gestes à poser. Le modèle informatique exige un pilote d'expérience pour être efficace. Et la méthode PEM évalue le passé. Alors quoi faire pour mieux appréhender l'avenir?

Communication et organisation, 30 | 2012 


\section{Les exigences de la publicité}

Revenons maintenant à la publicité. Il se dépense chaque année dans le monde quelque 400 milliards d'euros en publicité dont $80 \%$ en achat média.

L'industrie de la consommation et du marketing suit attentivement le développement de la psychologie du consommateur, ses habitudes d'achat, de détentes, de coups de cœur. Lorsque les annonceurs adoptent la publicité pour offrir leur produit ou service, ils disposent d'outils de mesures extrêmement efficaces et sophistiqués pour rejoindre un segment bien précis du public. Ils ont défini minutieusement leur cible et ils ont développé une connaissance fine de chacun des médias.

En fait, l'industrie de la publicité, c'est-à-dire les annonceurs, les agences de publicité et les médias, réalisent des études très poussées pour connaître les habitudes de consommation du public et ses habitudes d'utilisation des médias.

Ainsi, pour chaque quotidien et à l'intérieur de celui-ci pour chaque cahier, pour chaque rubrique, pour chaque chronique, on connaît le nombre exact, les caractéristiques sociodémographiques et les habitudes de consommation de chaque lecteur. Il en est de même pour chaque revue.

3 Pour la radio, on possède les mêmes données pour chaque station, par tranche de quart d'heures d'écoute. Et à la télévision, pour chacune des stations, c'est par émission que ces données sont présentées.

Pour la publicité d'affichage, on connaît pour chaque panneau le nombre et les caractéristiques sociodémographiques des automobilistes qui les croisent.

Pour que chaque euro soit bien investi, l'agence de l'annonceur cherchera le média et dans celui-ci la plage qui correspond le mieux à la stratégie définie et à la cible qu'il cherche à séduire ou à convaincre. Sans vouloir entrer dans trop de détails, la stratégie s'articule autour de notions comme la portée et la fréquence, c'est-à-dire est-ce que l'annonceur veut rejoindre le plus grand nombre d'un large public cible (la portée) ou veut-il répéter à un plus petit nombre le même message plusieurs fois (la fréquence). La stratégie média s'orchestrera ensuite en fonction du budget dont dispose l'annonceur.

Pour être en mesure d'arrêter ces choix, les spécialistes de la publicité disposent de plusieurs bases de données, une pour les quotidiens, une pour les journaux périodiques, une pour les magazines, une pour la radio, une pour la télévision et une pour l'affichage.

4 Ces bases de données sont complexes et chacune d'elles possède des informations précises qu'il faut apprendre à analyser. Il existe donc des spécialistes qui savent jongler avec cette masse de données. Ce sont les spécialistes de la stratégie média, ou du média planning selon l'expression utilisée en France.

8 Le site Internet du magazine français Action Commerciale, qui se spécialise dans les méthodes, les stratégies et les outils qui font vendre, définit le média planning comme suit :

« Media planning est l'ensemble des opérations qui visent à étudier puis à sélectionner les différents media, puis les différents supports, pour y réserver l'espace nécessaire à la diffusion du message publicitaire la plus efficace possible et au meilleur coût. On utilise pour cela de nombreux indicateurs tels que le coût au mille, le point de couverture brute, l'échelle d'économie, l'échelle de puissance, l'échelle de sélectivité, la L.D.P., la L.N.M.... ». 
50 Cette approche se divise en deux tâches distinctes, la planification média et le placement ou l'achat média. La planification média consiste :

- à choisir le média qui rejoint le mieux les objectifs et la cible visée

- à déterminer dans celui-ci l'espace le plus approprié pour la campagne,

- à décider du poids média, c'est-à-dire à fixer le nombre de passages requis pour influencer la cible

- à arrêter les périodes de diffusion

- à établir le calendrier de campagne

- à déterminer les budgets pour chaque média

- à mesurer les effets de la campagne

- Le placement média consiste :

- à s'assurer si les espaces souhaités sont disponibles

- à les réserver

- à négocier les coûts

- à assurer le contrôle et le suivi des espaces réservés

- à concilier les achats et les diffusions réelles des messages

51 Tous ces choix sont faits à partir de données précises. Une fois la stratégie média arrêtée, ce n'est ni le hasard ni l'intuition qui guident le spécialiste du média planning dans ses choix mais les chiffres et ceux-ci apportent des dimensions insoupçonnées. Au début des années 2000 au Québec, un magazine a fait paraître une page de publicité annonçant quelle était la revue féminine la plus lue, rejoignant ainsi plus de femmes que toute autre revue féminine. Toutefois, un magazine grand public a affirmé de son côté, qu'il rejoignait davantage de lectrices féminines que tout autre revue. Le planificateur média doit donc choisir s'il souhaite une revue féminine ou une revue grand public pour rejoindre les femmes. Or, ce sont les données factuelles qui permettent de trouver les bonnes réponses.

52 Il existe donc en publicité une méthode mathématique qui permet à l'annonceur et à son agence de publicité de faire des choix médias sélectifs extrêmement efficaces pour déterminer a priori les meilleurs canaux de diffusion pour rejoindre une cible dûment identifiée. Il s'agit donc là d'une méthode rationnelle et extrêmement utile pour construire une stratégie.

\section{Un outil délaissé en relations publiques}

Comment expliquer qu'une méthode extrêmement opérationnelle comme celle du média planning en publicité ne soit pas utilisée en relations publiques ? Les bases de données existent, les règles pour y avoir accès sont connues, leur efficacité est démontrée ? Tout se passe comme si, en relations publiques, l'important était de faire du bruit communicationnel et non d'attaquer une cible bien précise là où elle se trouve. Certes les montants investis en relations publiques sont si infimes face à la publicité qu'une opération mal ficelée n'occasionne pas de déboursés significatifs. Mais, si le résultat visé est d'influencer une cible, pourquoi alors ne pas prendre tous les moyens disponibles pour ce faire ? Il faut alors constater qu'en communication, la proposition de modèle est davantage inspirée par la formation et les préoccupations des chercheurs que par la recherche d'une meilleure façon de gérer la communication sur le plan professionnel et pratique. Et lorsque des méthodes ou modèles viennent de l'univers professionnel, il leur manque une certaine profondeur. 
54 dynamique socioculturelle de Moles ou le modèle génétique de Morin, aient reçu peu d'écho chez les praticiens du domaine des communications peut se comprendre car il s'agissait là de théorisation sur la réalité. Mais qu'une recette mathématique pour rejoindre la cible désignée soit écartée parce que les recherches dans les banques de données sont complexes et coûteuses, cela caractérise bien un métier qui se cherche une certaine maturité et parfois une certaine rigueur. Il est toutefois intéressant de réaliser qu'en relations publiques la modélisation intéresse davantage les scientifiques que les professionnels. Ce qui a comme corollaire que les modèles sont davantage théoriques que pratiques.

\section{BIBLIOGRAPHIE}

Covey Stephen R, The Seven Habits of Highly Effective People : Powerful Lessons in Personal Change, New York, Fireside Book, 1989.

Cutlip Scott M., Allen H. Center, Glen M.Broom, Effective Public Relations, Upper Saddle River : Prentice Hall, 1994.

Grunig James E. et Larissa A. Grunig, « Models of Public Relations and Communication, dans J. E. Grunig, Excellence in Public Relations and Communication Management, Hillsdale : Laurence Erlbaum Associates, 1992, pp. 285-325.

Katz E. et P.F. Lazarfeld P.F., Personal Influence, Glencoe : Free Press, 1955.

Kugler Marianne, Des campagnes de communication réussies : 43 études de cas primes, Sainte-Foy : Presses de l'Université du Québec, 2004.

Laswell H.D., « The structure and function of communication in society», in Bryson, (ed.), The Communication of Ideas, New York : Harper and Brothers, 1948.

Lavigne Alain, « La communication institutionnelle vue en tant que six grands réseaux institutionnels», Communication et organisation, vol. 21, pp. 154-173, 2002.

Lougovoy Constantin, Huisman Denis, Traité de relations publiques, Paris, PUF, 1981.

Marston John E., The Nature of Public Relations, N.Y. : McGraw-Hill, 1963.

Moles Abraham, Sociodynamique de la culture, Paris, LaHaye, Mouton, 1967.

Monod Jacques, avec la collaboration de François Jacob, Le hasard et la nécessité, Paris, Éditions du Seuil, 1970, 197 p.

Morin Edgar, La méthode 3 : La connaissance de la connaissance, Paris, Le Seuil, 1986.

Mucchielli A, 2000, L'art d'influencer, Paris : Armand Colin.

Noëlle-Neumann E. 1974, « The Spiral of Silence : A Theory of Public Opinion», Journal of Communication, vol. 25, 1974, pp. 43-51.

Philibert Jean-René, Modèle de définition des actions prioritaires en relations publiques, Mémoire de maîtrise, Département d'information et de communication, Université Laval, 2005. 
Shannon Claude E. et Warren Weaver, The Mathematical Theory of Communication, Urbana : University of Illinois Press, 1949.

Willett Gilles (sous la direction de), La communication modélisée, Montréal : Éditions du Renouveau pédagogique, 1992.

\section{NOTES}

1. Shannon Claude E. et Warren Weaver, The Mathematical Theory of Communication, Urbana: University of Illinois Press, 1949.

2. Laswell H.D., " The structure and function of communication in society», in Bryson, (ed.), The Communication of Ideas, New York : Harper and Brothers, 1948.

3. Katz E. et P.F. Lazarfeld, Personal Influence, Glencoe : Free Pres, 1955.

4. Moles Abraham, Sociodynamique de la culture, Paris, LaHaye, Mouton, 1967.

5. Willett Gilles (sous la direction de), 1992, La communication modélisée, Montréal : Éditions du Renouveau pédagogique.

6. MonodJacques, avec la collaboration de François Jacob, 1970, Le hasard et la nécessité, Paris, Éditions du Seuil.

7. Morin Edgar, La Méthode, tome 1 à 5

8. Op.cit, p. 24 à 35 .

9. Lougovoy Constantin et Denis Huisman, 1981, Traité de relations publiques, Paris, PUF

10. Cutlip Scott M., Allen H. Center, Glen M.Broom, 1994, Effective Public Relations, Upper Saddle River : Prentice Hall.

11. Noëlle-Neumann E. 1974, "The Spiral of Silence : A Theory of Public Opinion», Journal of Communication, vol. 25, pp. 43-51.

12. Marston John E., 1963, The Nature of Public Relations, N.Y. : McGraw-Hill.

13. Grunig James E. et Larissa A. Grunig, 1992, « Models of Public Relations and Communication, dans J. E. Grunig, Excellence in Public Relations and Communication Management, Hillsdale : Laurence Erlbaum Associates, pp. 285-325.

14. Kugler Marianne, 2004, Des campagnes de communication réussies : 43 études de cas primes, SainteFoy : Presses de l'Université du Québec.

15. Lavigne Alain, 2002, « La communication institutionnelle vue en tant que six grands réseaux institutionnels», Communication et organisation, vol. 21, pp. 154-173.

16. Mucchielli A, 2000, L'art d'influencer, Paris : Armand Colin.

17. Philibert Jean-René, 2005, Modèle de définition des actions prioritaires en relations publiques, Mémoire de maîtrise, Département d'information et de communication, Université Laval.

18. Op.cit, p. 8.

19. Covey Stephen R, 1989, The Seven Habits of Highly Effective People : Powerful Lessons in Personal Change, New York, Fireside Book., p. 151.

20. Op. cit p. 54.

21. Op cit, p. 101. 


\section{RÉSUMÉS}

Les modèles qu'utilisent les différentes disciplines de la connaissance humaine sont empreints d'effets de mode. En communication, certains modèles s'imposent, comme celui de Shannon et Wiener, et d'autres ont une courte vie comme celui de Moles (la dynamique socio-culturelle) et Morin (le modèle génétique). Les relations publiques ont créé peu de modèles qui leur soient propres, parmi lesquels le modèle RACE. Mais elles ont aussi été hermétiques à des modèles très efficaces, comme les méthodes du média planning.

Models used by the different disciplines dealing with human knowledge are biased by mode effects. In the field of communication, some models can't be ignored, as it is the case of Shannon and Wiener's, yet others have a limited lifetime, such as Moles' (socio-cultural dynamics) and Morin's (the genetic model). Public relations are at the origin of few models, among which the RACE model. Besides, public relations were also hermetic to very efficient models, such as the media planning methods.

\section{INDEX}

Keywords : model, public relations, advertising

Mots-clés : relations publiques, publicité, média planning, modèle 